\title{
The comparative effect of exposure to various risk factors on the risk of hyperuricaemia: diet has a weak causal effect
}

Ruth K. G. Topless ${ }^{1 \dagger}$, Tanya J. Major ${ }^{1 \dagger}$, Jose C. Florez ${ }^{2,3,4}$, Joel N. Hirschhorn ${ }^{2,5,6}$, Murray Cadzow ${ }^{1}$, Nicola Dalbeth ${ }^{7}$, Lisa K. Stamp ${ }^{8}$, Philip L. Wilcox ${ }^{9}$, Richard J. Reynolds ${ }^{10}$, Joanne B. Cole $2,3,5+$ and Tony R. Merriman ${ }^{1,10^{*+}}$ (D)

\begin{abstract}
Background: Prevention of hyperuricaemia (HU) is critical to the prevention of gout. Understanding causal relationships and relative contributions of various risk factors to hyperuricemia is therefore important in the prevention of gout. Here, we use attributable fraction to compare the relative contribution of genetic, dietary, urate-lowering therapy (ULT) and other exposures to HU. We use Mendelian randomisation to test for the causality of diet in urate levels.

Methods: Four European-ancestry sample sets, three from the general population $(n=419,060)$ and one of people with gout $(n=6781$ ) were derived from the Database of Genotypes and Phenotypes (ARIC, FHS, CARDIA, CHS) and UK Biobank. Dichotomised exposures to diet, genetic risk variants, BMI, alcohol, diuretic treatment, sex and age were used to calculate adjusted population and average attributable fractions (PAF/AAF) for HU ( $\geq 0.42 \mathrm{mmol} / \mathrm{L}$ [ $\geq 7 \mathrm{mg} / \mathrm{dL}]$ ). Exposure to ULT was also assessed in the gout cohort. Two sample Mendelian randomisation was done in the UK Biobank using dietary patternassociated genetic variants as exposure and serum urate levels as outcome.
\end{abstract}

Results: Adherence to dietary recommendations, BMI $\left(<25 \mathrm{~kg} / \mathrm{m}^{2}\right)$, and absence of the SLC2A9 rs 12498742 urate-raising allele produced PAFs for HU of 20 to $24 \%, 59$ to $69 \%$, and 57 to $64 \%$, respectively, in the three non-gout cohorts. In the gout cohort, diet, BMI, SLC2A9 rs 12498742 and ULT PAFs for HU were 12\%, 49\%, 48\%, and 63\%, respectively. Mendelian randomisation demonstrated weak causal effects of four dietary habits on serum urate levels (e.g. preferentially drinking skim milk increased urate, $\beta=0.047 \mathrm{mmol} / \mathrm{L}, P=3.78 \times 10^{-8}$ ). These effects were mediated by $B M \mathrm{l}$, and they were not significant $(P \geq 0.06)$ in multivariable models assessing the BMI-independent effect of diet on urate.

Conclusions: Diet has a relatively minor role in determining serum urate levels and HU. In gout, the use of ULT was the largest attributable fraction tested for $\mathrm{HU}$.

Keywords: Hyperuricemia, Gout, Risk factor, Population attributable fraction, Variance, Genetic polymorphism, Diet, Mendelian randomisation

\footnotetext{
*Correspondence: tony.merriman@otago.ac.nz

${ }^{\dagger}$ Ruth K. G. Topless, Tanya J. Major, Joanne B. Cole and Tony R. Merriman contributed equally to this work.

'Department of Biochemistry, University of Otago, Dunedin, New Zealand

${ }^{10}$ Division of Clinical Immunology and Rheumatology, University of Alabama Birmingham, Birmingham, AL, USA

Full list of author information is available at the end of the article
}

(C) The Author(s). 2021 Open Access This article is licensed under a Creative Commons Attribution 4.0 International License, which permits use, sharing, adaptation, distribution and reproduction in any medium or format, as long as you give appropriate credit to the original author(s) and the source, provide a link to the Creative Commons licence, and indicate if changes were made. The images or other third party material in this article are included in the article's Creative Commons. licence, unless indicated otherwise in a credit line to the material. If material is not included in the article's Creative Commons licence and your intended use is not permitted by statutory regulation or exceeds the permitted use, you will need to obtain permission directly from the copyright holder. To view a copy of this licence, visit http://creativecommons.org/licenses/by/4.0/ The Creative Commons Public Domain Dedication waiver (http://creativecommons.org/publicdomain/zero/1.0/) applies to the data made available in this article, unless otherwise stated in a credit line to the data. 


\section{Introduction}

Hundreds of genetic variants are associated with serum urate levels [1-3], and observational studies have associated individual dietary factors (e.g. alcohol, sugarsweetened beverages, coffee, red-meat consumption [4$8]$ ) and overall eating habits $[9,10]$ with urate levels, along with other environmental (e.g. diuretic use [11, 12]) and endogenous factors (e.g. age and sex [13]). Understanding the importance of risk factors and their causal relationship (if any) with HU is critical in developing strategies for the prevention of $\mathrm{HU}$ and gout. However, addressing causality is challenging. Observational, longitudinal or migratory studies, and temporal correlations can only be regarded as hypothesis-generating owing to the intractable issue of unmeasured confounding. Any attempts to draw conclusions with respect to causality, including using causal language/ inferences, even from an accumulation of studies, misrepresents the evidence [14]. In this context, we note that gout is a multi-stage process beginning with HU, progressing to deposition of monosodium urate crystals and culminating in an innate immune response to crystals [15]. Not all people with HU develop gout [16], so HU and the progression from $\mathrm{HU}$ to gout should not be conflated when considering possible causal risk factors.

The gold standard for testing an exposure for a causal role is the randomised clinical trial (RCT). This approach has demonstrated causality for dissolved sugar (sugarsweetened beverages) in raising urate levels [17-19]. However, for the majority of suspected causal exposures an RCT is not possible. Mendelian randomisation (MR) exploits the natural randomisation of alleles causal for a particular exposure and is analogous to a RCT. Several MR studies have shown a small causal effect of BMI on urate levels $(0.0045$ to $0.010 \mathrm{mmol} / \mathrm{L}$ [0.075-0.17 mg/dL] increase in serum urate per unit increase in genetically determined BMI [20-22]). Dietary preferences have a heritable component and genetic associations have been reported [23-25]. Mendelian randomisation in the UK Biobank, using genetic variants associated with dietary patterns has demonstrated that a 'healthful' versus 'unhealthful' dietary pattern is not strongly causal for coronary heart disease or type 2 diabetes, despite diet being strongly correlated with these diseases [26].

Using the widely applied approach of decomposing variance, where the sum of multiple risk factors included in a model is constrained to $100 \%$, overall diet contributed $\leq 0.3 \%$ of variance in urate levels, substantially less than the $23.9 \%$ explained by inherited common genetic variants [10]. Why so little variance is explained is unclear, but one possible reason is that overall diet, which comprises some foods associated with increased urate and some foods associated with decreased urate, does not play a strong causal role. This possibility is supported by a RCT that reported a small $0.021 \mathrm{mmol} / \mathrm{L}(0.35 \mathrm{mg} / \mathrm{dL})$ reduction in urate levels in people following the Dietary Approaches to Stop Hypertension (DASH) diet compared to those on a 'typical' US diet [27]. Another RCT comparing the Mediterranean diet to a 'prudent Westernised diet' reported a small reduction in serum urate levels $(0.010 \mathrm{mmol} / \mathrm{L}[0.17$ $\mathrm{mg} / \mathrm{dL}]$ ) over 5 years [28]. Another possible explanation postulated in ref. [9] for the small amount of variance explained is low variability in diet within the US cohorts used in [10]. However, differences in diet between men and women, across age groups, socioeconomic status, ethnicity and BMI strata in the US have been reported [29]to observe these differences there must be variability in the diet.

Population attributable fraction (PAF) is the proportion of cases for an outcome within a population that can be attributed to a given risk factor, incorporating both the prevalence and the effect size of the exposure [30]. The sum of PAFs for multiple risk factors for a single condition is not constrained to $100 \%$, because an outcome can have multiple risk pathways populationwide. Using the Third National Health and Nutrition Examination Survey PAFs were reported of $44 \%$ for being overweight or obese (implying that $44 \%$ of HU would be prevented if the entire population had BMI $<25 \mathrm{~kg} /$ $\mathrm{m}^{2}$ ) and $9 \%$ for non-adherence to a DASH-style diet [9]. Variances explained were $8.3 \%$ and $0.1 \%$, respectively [9], indicating the two methods agree as to which exposure has a greater impact.

Our first aim was to use attributable fraction to compare the contributions to $\mathrm{HU}$ of various genetic, environmental and endogenous risk factors, including lack of use of urate-lowering therapy. The second aim was to use MR to test for a causal role of diet in determining urate levels.

\section{Participants and methods \\ Participants and data collection}

The attributable fraction analysis included four distinct cohorts of European ancestry (Table 1) - cohorts 1 to 3 are population-based and the fourth cohort is comprised entirely of people with gout.

Cohort 1 comprised 14,247 participants of European ancestry from the US population-7342 from the Atherosclerosis Risk in Communities (ARIC) Study, 1314 from the Coronary Artery Risk Development in Young Adults (CARDIA) Study, 2513 from the Cardiovascular Health Study (CHS) and 3078 from the Framingham Heart Study (FHS). These numbers exclude people without serum urate measurements or genome-wide genotypes, along with individuals aged under 18 years, people with kidney disease or gout and those taking uratelowering therapy. People who answered less than $10 \%$ of the food frequency survey, those whose estimated average daily calorie intake was less than $600 \mathrm{kcal} /$ day or 


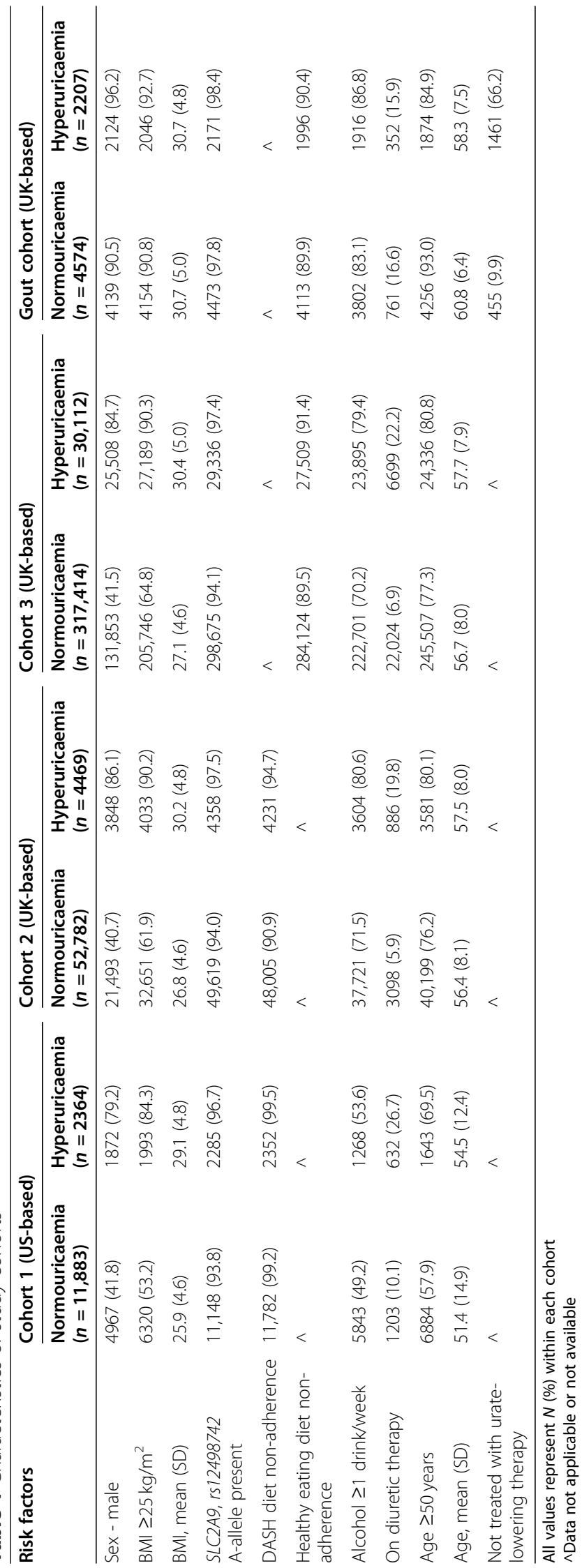


greater than $4200 \mathrm{kcal} /$ day and those whose questionnaire answers were deemed unreliable by the study interviewer at recruitment were also excluded. For ARIC, one person from each first degree-related pair was excluded.

Cohort 2, cohort 3 and the gout cohort were sourced from the UK Biobank resource. Subjects of European ancestry and who had urate measures and genotypes available were included in the analysis. Relatives with kinship coefficients $>0.177$ were removed, and one person from each relationship was kept, with a preference for keeping gout-affected participants. Those who self-reported having kidney disease were also removed. The gout cohort comprised people who self-reported having gout at visit 0 or were being treated with urate-lowering therapy $(n=$ 6781) [31], this case definition has been validated [31, 32]. Cohort 2 consisted of UK Biobank participants who answered a 24-h dietary recall questionnaire during the assessment visit $(n=57,251)$ and cohort 3 consisted of the remaining UK Biobank subjects who answered a reduced food frequency questionnaire $(n=347,526)$. We excluded, from cohort 2, subjects who had energy intakes in excess of $18,000 \mathrm{~kJ}$ for females and $20,000 \mathrm{~kJ}$ for males based on their 24-h dietary recall data, those who had unreliable dietary data as flagged by the recruiter, or subjects not eating normally due to illness or fasting. No additional exclusions were applied for cohort 3 or the gout cohort. Participants used for the MR analysis were also from the UK Biobank, and the MR cohort has been described previously [26].

Collection of dietary and serum urate data are described in the Supplemental material. Collection of genetic data for the ARIC, FHS, CHS and CARDIA cohorts is described in ref. [10] and for the UK Biobank in [33].

\section{Data dichotomisation}

To calculate a PAF, all exposure and outcome variables must be dichotomous. The outcome for this study was $\mathrm{HU}$, defined as serum urate $\geq 0.42 \mathrm{mmol} / \mathrm{L}$ [ $\geq 7 \mathrm{mg} / \mathrm{dL}$ ] for men and women [34]. For the genetic exposures, HU risk alleles were defined as urate-increasing under a dominant model [35]. Determination of dichotomised dietary exposures is described in the Supplemental material. Alcohol exposure was defined as $>1$ drink per week, being overweight/obese as BMI $\geq 25 \mathrm{~kg} / \mathrm{m}^{2}$, age was dichotomised as $\geq 50$ versus $<50$ years partly in order to capture menopause as a risk factor in women and diuretic use either self-reporting or not self-reporting diuretic intake-these variables were the same as those for which PAR estimates were calculated in the Third National Health and Nutrition Examination Survey in ref. [9] and for age. In the gout cohort, self-reported treatment with urate-lowering therapies, allopurinol $(n=4841)$, probenecid $(n=3)$ and sulphinpyrazone $(n=21) \quad$ (the only three urate-lowering medications for which baseline medication data were available) was a dichotomised exposure variable.

\section{Statistical analysis}

All analyses were performed using R v3.6.1 in RStudio 1.2.5019. For the various exposures, the PAF calculation was (frequency of exposure in cases) $\times\left(\mathrm{OR}_{\text {Exposure }}-1\right) /$ $\mathrm{OR}_{\text {Exposure }}$ ) [36]. Odds ratios for the risk of $\mathrm{HU}$ for these exposures were calculated in a logistic regression multivariable model including all other environmental and endogenous exposure variables and SLC2A9 rs12498742 genotype-this variant was chosen for individual focus because of its large effect on serum urate levels [35]. For the percent variance explained analysis (Table 3), effect sizes $(\beta)$ on serum urate levels for the same exposures were calculated in a linear regression multivariable model including all other environmental and endogenous exposure variables and SLC2A9 rs12498742 genotype. Average attributable fractions (AAFs), adjusted for all other exposure variables and rs12498742 genotype (Table 2) were calculated using a multivariable model in the $\mathrm{R}$ function averageAF [36], described in more detail in Supplemental material. Age was removed as it did not confer risk in the gout cohort. In general, AAFs are lower than PAFs calculated from the same data (as consistently observed here) and it has been proposed that they reflect the most plausible/reliable result across the many different methods of calculating attributable fractions [37].

For genetic variants in Table S2, PAFs (and odds ratios) were calculated per allele under an additive model using the method of Rockhill et al. [36] ((frequency risk allele in cases $\left.) \times\left(\left(\mathrm{OR}_{\text {RiskAllele }}-1\right) / \mathrm{OR}_{\text {RiskAllele }}\right)\right)$. However, PAFs (and odds ratios) in Tables 2, S1 and Fig. 1 were calculated under a dichotomised (dominant) model with the exposed group defined as those with one or more risk allele and were adjusted for dichotomised age, sex, BMI, diet, alcohol and diuretic exposure risks. All AAF analyses used a dominant model for SNP analysis because risk was dichotomised.

\section{Mendelian randomisation}

Two-sample MR using the MendelianRandomization $\mathrm{R}$ package [38] was tested for a causal role of dietary habits in determining urate levels. GWAS summary statistics for urate levels in Europeans were obtained from ref. [3] comprising 288,649 individuals; 101 loci with nonambiguous lead SNPs where no strand assignation issues could have arisen by GWAS which can occur with A/T and $\mathrm{G} / \mathrm{C}$ variants where the alternative allele is the same as the potential strand-flipped allele. This conservative approach was taken in order to harmonise effect alleles from a meta-analysis of multiple studies using multiple imputation panels. In order to test that this QC did not 
Table 2 Population attributable and average attributable fractions for $\mathrm{HU}$ risk exposures

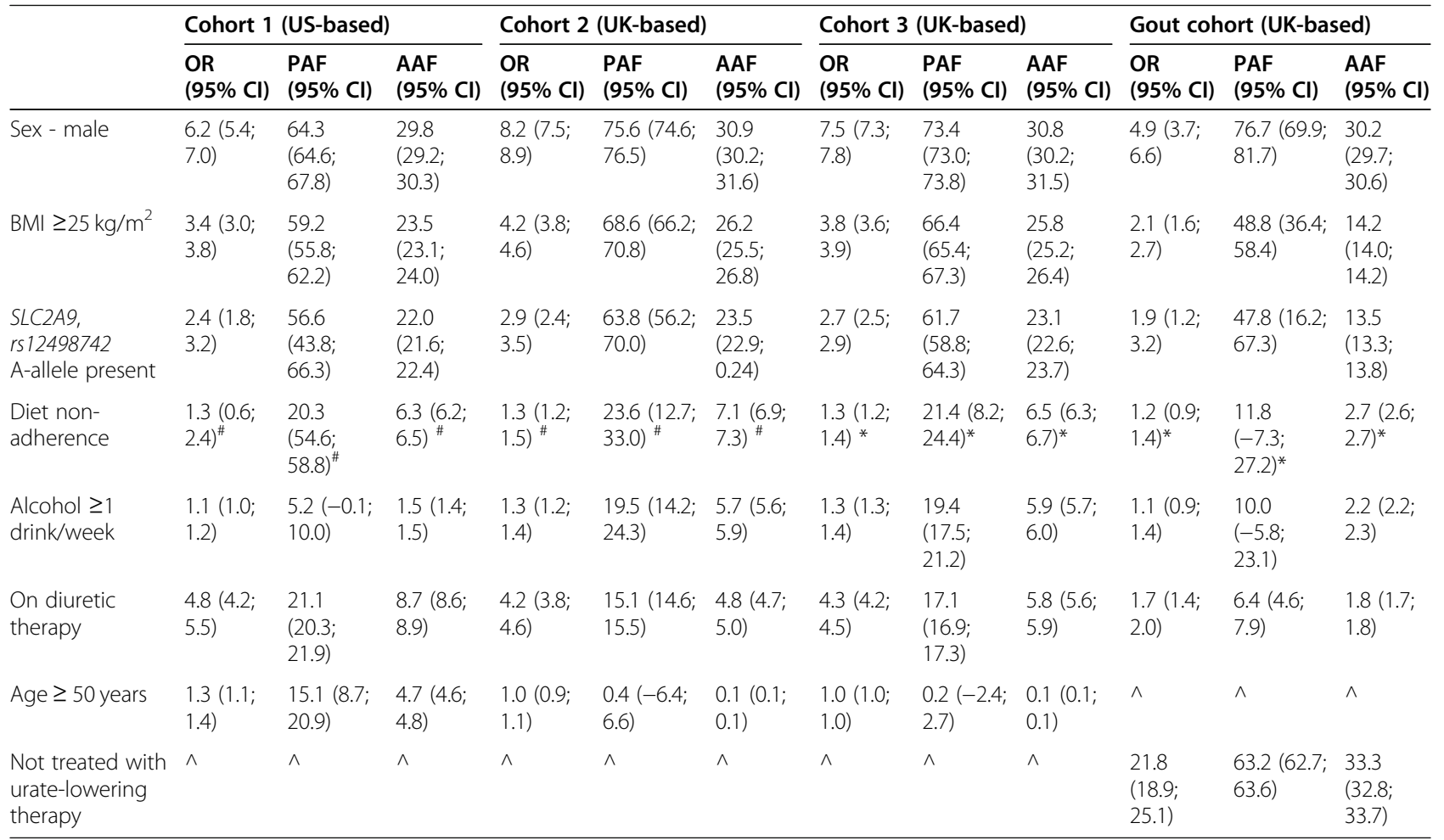

${ }^{\#}$ Non-adherence to DASH guidelines; ${ }^{*}$ Non-adherence to the Harvard Healthy Eating Pyramid guidelines; $\wedge$ Data not applicable or variable is not a risk factor in this group

All analyses are adjusted in a multivariable model for the other risk factors. OR calculations were done in a logistic regression model. For the gout cohort in a model including age, the OR was $0.5(0.4 ; 0.6)$. For SLC2A9 rs 12498742, PAF and AAF were calculated under a dominant model for the A-allele

influence the results, we conducted a sensitivity analysis and repeated the primary Mendelian randomisation with the inclusion of all ambiguous SNPs (Figure S1)-the results were in high concordance. GWAS data for dietary habits were obtained from a study of 455,146 individuals of European ancestry from the UK Biobank [26]. The latter were derived from consumption patterns for 47 single foods asked about in the reduced food frequency questionnaire or 40 principal component-derived dietary patterns with $\geq 3$ non-ambiguous genome-wide significant index SNPs reported in ref. [26] that were also present in the serum urate GWAS summary statistics [3]. Independence of loci was based on the distancebased pruning approach within the source GWAS [3, 26] - loci had to be $>500 \mathrm{~kb}$ apart and if there were two in a $1 \mathrm{Mb}$ interval independence was established by plotting and visualisation of inter-marker linkage disequilibrium. The MR was performed using three methods available in the MendelianRandomization R packageinverse-variance-weighted meta-analysis [39], MR Egger (enables detection of pleiotropy [40]) and weighted median (robust to pleiotropy [41]). Significance thresholds were set at $P<0.05 / 87\left(5.8 \times 10^{-4}\right)$ for the inversevariance-weighted analysis, $P<0.05$ for the weighted median analysis and intercept $P>0.05$ for the MR Egger analysis. Our strategy was to identify causal effects from the inverse-variance-weighted analysis corrected for multiple testing followed by sensitivity analysis by weighted median and MR Egger to test the robustness of the results. All three significance thresholds had to be met for a dietary habit to be considered causal. Multivariable MR was conducted to investigate the possible upstream impact of BMI as a common causal link between dietary patterns and serum urate levels using the likelihood-based method on summary data as described by Burgess and Thompson [42], as implemented in the MendelianRandomization $\mathrm{R}$ package. This technique uses pleiotropic genetic variants to estimate the direct effect of multiple exposures on an outcome (e.g. BMI and diet on serum urate), with causal estimates representing the independent causal effect of each exposure on the outcome, not operating through the other exposure included in the analysis.

\section{Results}

Population and average attributable fractions

Male sex had the largest PAF and AAF (64.3 to $75.6 \%$, and 29.8 to $30.9 \%$, respectively, in cohorts 1 to 3 ), being overweight or obese had the second largest measures $(\mathrm{PAF}=59.2$ to $68.6 \%, \quad \mathrm{AAF}=23.5$ to $26.2 \%)$, and 


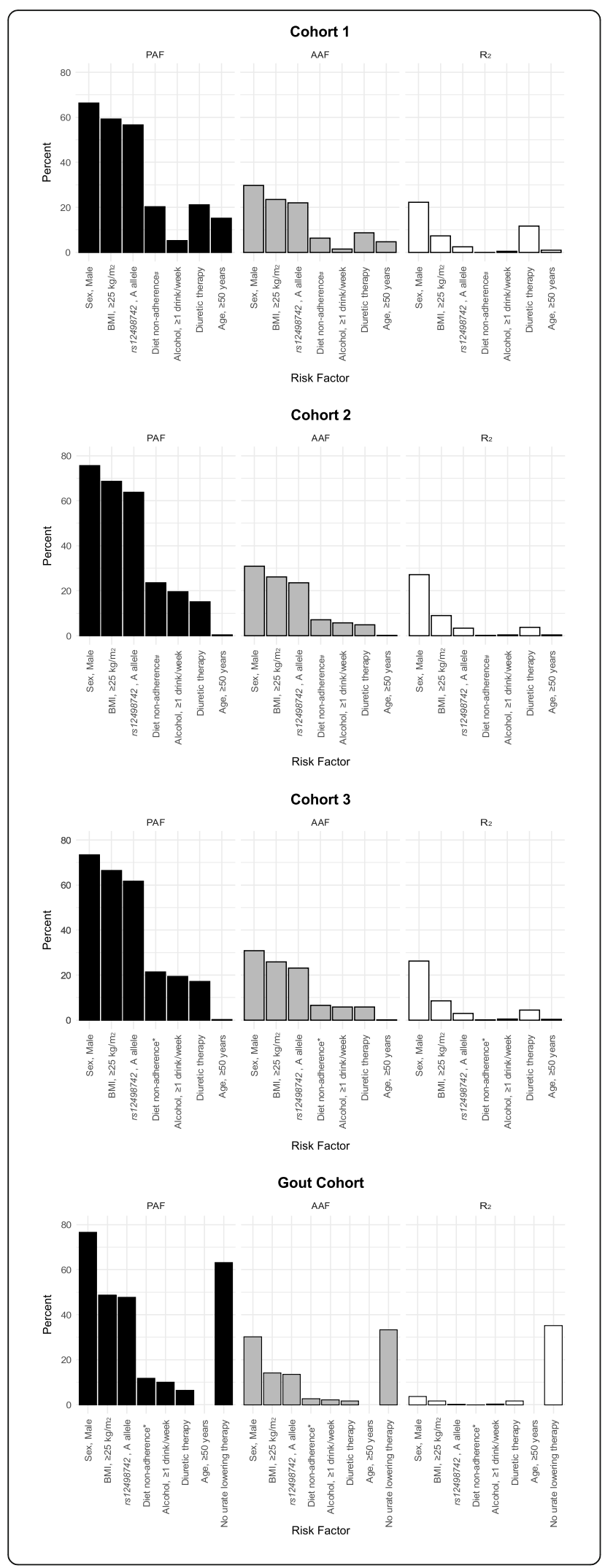

Fig. 1 Population attributable fraction, average attributable fraction and variance explained for environmental and endogenous risk exposures for hyperuricaemia or serum urate levels. PAF - population attributable fraction; AAF - average attributable fraction; $R^{2}$ - partial $R^{2}$ value $\left(R_{\mathrm{B}}^{2}\right)$ converted to a percentage $\left(R^{2} * 100\right)$. Population attributable fraction and average attributable fraction values relate to hyperuricemia as a dichotomous variable where for the SLC2A9 rs 12498742 A-allele PAF and AAF was calculated under a dominant model; $R^{2}$ relates to serum urate as a continuous variable. All risk factors are dichotomous. All analyses are adjusted in a multivariable model for the other risk factors. "Non-adherence to DASH guidelines; *Non-adherence to the Harvard Healthy Eating Pyramid guidelines. Missing bars in Fig. 1d reflect age not being a risk factor in this group

inheriting the $S L C 2 A 9$ rs12498742 A-allele had the third largest effect ( $\mathrm{PAF}=56.6$ to $63.8 \%, \mathrm{AAF}=22.0$ to $23.5 \%$ ). All other factors had PAFs $<25 \%$ and AAFs $<10 \%$ (Table 2). Cohorts 2 and 3 had the same ranking of risk factors $($ sex $>$ BMI $>$ rs12498742 $>$ diet adherence $>$ alcohol consumption $>$ diuretic therapy $>$ age), while cohort 1 ranked the same three risk factors first (sex $>\mathrm{BMI}>$ rs12498742), before differing in rankings of the remaining four risk factors (diuretic therapy $>$ diet adherence $>$ age $>$ alcohol consumption) (Fig. 1).

In the gout cohort, lack of treatment with uratelowering therapy had the second largest PAF of $63.2 \%$, after sex (76.7\%) (Table 2). The PAFs for BMI and SLC2A9 rs12498742 were lower than for the non-gout cohorts (57 to $69 \%$ in non-gout and 48 to $49 \%$ in gout), and non-adherence to the Healthy Eating Pyramid guidelines was $21.4 \%$ in non-gout (cohort 3) and $11.8 \%$ in gout. Average attributable fraction values showed a similar trend (Fig. 1).

In sex-stratified analysis in the non-gout cohorts, PAFs for rs 12498742 were 77.7 to $96.4 \%$ in women compared to 49.2 to $58.9 \%$ in men (Table S1). Alcohol was not a risk factor in women across all cohorts, nor age in men in cohorts 2 and 3, and gout (Table S1; 95\% CI encompassed 1.0). The lower age limit for recruitment into the UK Biobank, which these two cohorts were derived from, was 40 years, which may have influenced the calculation.

In the non-gout cohorts, of 30 genetically-independent serum urate-associated genetic variants chosen as having the top effects by GWAS [33] evaluated (Table S2), the SLC2A9 rs12498742 variant was the largest, with PAFs ranging from 28.5 to $32.1 \%$ and AAFs from 22.0 to $23.5 \%$. For comparative purposes, we summed the PAFs for genetic variants, assuming that the variants act independently of each other to influence the risk of $\mathrm{HU}$, with the individual PAFs summing to $>141 \%$ (cohort 1 was $146.2 \%$, cohort 2 was $143.7 \%$, cohort 3 was $141.3 \%$ ). Summing the AAFs (equivalent to the summing of PAFs, above) resulted in all three cohorts having a summed AAF over $87 \%$ (cohort 1 was $97.9 \%$, cohort 2 was $87.1 \%$, 
cohort 3 was 101.6\%). Summed attributable fractions for genetic variants for $\mathrm{HU}$ were considerably lower for the gout cohort (PAF was $77.6 \%$ and AAF was $44.0 \%$ ), possibly reflecting selection (collider) bias.

\section{Percent variance explained for serum urate levels}

In the non-gout cohorts, sex had the most percent variance explained (22 to 27\%) (Table 3). The dichotomised BMI exposure was consistently 7 to $9 \%$, with diuretic exposure accounting for $12 \%$ variance in the US-based cohort and $4-5 \%$ in the UK-derived cohorts. The diet estimate was $\leq 0.1 \%$ and $S L C 2 A 9$ was $2-3 \%$, similar to our previous report [10]. The gout cohort included urate-lowering therapy exposure in the model, with exposure accounting for the largest proportion (35\%) of variance, approximately 10 -fold more than any other variable. The use of percent variance explained produced a broadly similar ranking order of risk factors to the PAF and AAF analyses across all four cohorts (Fig. 1).

\section{Mendelian randomisation}

Five of the 87 single foods and principal componentderived dietary-associated habits [26] provided evidence of a causal effect (IVW $P<0.05 / 87\left(5.7 \times 10^{-4}\right)$ ) on urate levels by inverse-variance-weighted MR (Table S3). All five of these dietary habits also had no evidence for an intercept significantly different from zero in the MR Egger analysis (all $P>0.05$ ) indicating no evidence for directional (horizontal) pleiotropy. Four of these dietary habits provided evidence for a causal role $(P<0.05)$ and yielded similar effect sizes in the weighted median analysis (Table 4). Two of these causal effects were with dairyrelated dietary habits (preferentially drinking skim milk and preferentially drinking milk with a higher fat content), and the other two causal effects were for consuming tub margarine and daily dried fruit consumption.

Of the 39 genetic variants that comprised the four dietary-associated habits, 21 are associated with metabolic traits (http://www.type2diabetesgenetics.org/ [accessed: 2nd June 2020]) and/or traits available in the UKBiobank PheWeb (http://pheweb.sph.umich.edu:5000 [accessed: June 2, 2020]), including 16 specifically associated with BMI or a related body fat trait (Table S4). To test the possibility that the causal association between these four dietary habits and urate levels is due to BMI as a common upstream cause (e.g. change in dietary habits due to weight-loss advice), we applied multivariable MR using the same individual level UK Biobank dataset described in ref. [26]. For all four dietary patterns, including BMI in the multivariable analysis resulted in no evidence for a causal effect $(P \geq 0.06)$, BMI showing a causal relationship with urate levels independent of the dietary habit (Fig. 2). Bidirectional MR between BMI and each of the four dietary habits where, by inverse varianceweighted meta-analysis MR BMI was tested for a causal effect on the dietary habits and each of the dietary habits was tested for a causal effect on BMI, conducted to confirm whether BMI is a common upstream cause of dietary habits, provided evidence in both directions $(P \leq 4.4 \times$ $10^{-18}$ for BMI to dietary habit, $P \leq 9.4 \times 10^{-4}$ for dietary habit to BMI) - a situation termed "correlated pleiotropy" [43] - except in the margarine analysis for the BMI to dietary habit analysis $(P=0.24)$ although there was evidence for the dietary habit to BMI analysis $\left(P=8.4 \times 10^{-61}\right)$. This indicates that $\mathrm{BMI}$ and the four dietary habits are strongly correlated traits or work through a shared pathway and that the four dietary habits have no effect on urate levels independent of BMI.

Table 3 Associations of dichotomised risk variables with serum urate levels (including percent variance explained)

\begin{tabular}{|c|c|c|c|c|c|c|c|c|}
\hline \multirow[t]{2}{*}{ Risk factor } & \multicolumn{2}{|l|}{ Cohort 1 (US-based) } & \multicolumn{2}{|l|}{ Cohort 2 (UK-based) } & \multicolumn{2}{|c|}{ Cohort 3 (UK-based) } & \multicolumn{2}{|c|}{ Gout cohort (UK-based) } \\
\hline & Beta $(95 \% \mathrm{Cl})$ & $R^{2}(\%)$ & Beta $(95 \% \mathrm{Cl})$ & $R^{2}(\%)$ & Beta $(95 \% \mathrm{Cl})$ & $R^{2}(\%)$ & Beta $(95 \% \mathrm{Cl})$ & $R^{2}(\%)$ \\
\hline Sex-male & $0.078(0.076 ; 0.080)$ & 22.4 & $0.076(0.075 ; 0.077)$ & 27.2 & $0.075(0.075 ; 0.076)$ & 25.9 & $0.062(0.055 ; 0.070)$ & 3.7 \\
\hline $\mathrm{BMI} \geq 25 \mathrm{~kg} / \mathrm{m}^{2}$ & $0.041(0.038 ; 0.043)$ & 7.3 & $0.039(0.038 ; 0.041)$ & 9.0 & $0.040(0.039 ; 0.040)$ & 8.5 & $0.032(0.025 ; 0.039)$ & 1.8 \\
\hline $\begin{array}{l}\text { SLC2A9, rs } 12498742 \\
\text { A-allele present }\end{array}$ & $0.044(0.039 ; 0.049)$ & 2.5 & $0.048(0.046 ; 0.050)$ & 3.4 & $0.047(0.046 ; 0.047)$ & 3.0 & $0.026(0.009 ; 0.043)$ & 0.02 \\
\hline Diet non-adherence & $0.008(-0.006 ; 0.021)^{\#}$ & 0.01 & $0.008(0.006 ; 0.010)^{\#}$ & 0.1 & $0.005(0.004 ; 0.005)^{*}$ & 0.1 & $0.004(-0.003 ; 0.010)^{*}$ & 0.01 \\
\hline Alcohol $\geq 1 \mathrm{drink} /$ week & $0.004(0.002 ; 0.007)$ & 0.2 & $0.008(0.007 ; 0.009)$ & 0.4 & $0.009(0.008 ; 0.009)$ & 0.5 & $0.005(-0.001 ; 0.010)$ & 0.3 \\
\hline On diuretic therapy & $0.058(0.054 ; 0.061)$ & 11.7 & $0.047(0.045 ; 0.049)$ & 3.7 & $0.049(0.048 ; 0.050)$ & 4.5 & 0.029 (0.029; 0.035) & 1.7 \\
\hline Age $\geq 50$ years & $0.014(0.012 ; 0.017)$ & 0.9 & $0.009(0.008 ; 0.010)$ & 0.4 & $0.009(0.009 ; 0.010)$ & 0.4 & $\wedge$ & $\wedge$ \\
\hline $\begin{array}{l}\text { Not treated with } \\
\text { urate-lowering therapy }\end{array}$ & $\wedge$ & $\wedge$ & $\wedge$ & $\wedge$ & $\wedge$ & $\wedge$ & $0.133(0.129 ; 0.138)$ & 35.2 \\
\hline
\end{tabular}

\#Non-adherence to DASH guidelines; *Non-adherence to the Harvard Healthy Eating Pyramid guidelines; $\wedge$ Data not applicable or variable is not a risk factor in this group

Beta values represent urate change in $\mathrm{mmol} / \mathrm{L}$ between risk groups. $R^{2}$ - partial $R^{2}$ value $\left(R_{\mathrm{B}}^{2}\right)$ converted to a percentage $\left(R^{2} * 100\right)$. All analyses are adjusted in a multivariable linear regression model for the other risk factors. Risk for SLC2A9 rs 12498742 was calculated under a dominant model. For the Gout cohort in a model including age, the beta was $-0.036(-0.043 ;-0.029)$ and the $R^{2}$ was 1.7 
Table 4 Causal effects of dietary habits on serum urate: significant Mendelian randomisation results only

\begin{tabular}{|c|c|c|c|c|c|c|c|c|}
\hline \multirow[t]{2}{*}{ Dietary pattern description } & \multirow{2}{*}{$\begin{array}{l}\text { SNP } \\
\text { number }\end{array}$} & \multicolumn{3}{|c|}{ Inverse-variance-weighted MR } & \multicolumn{2}{|l|}{ MR Egger } & \multicolumn{2}{|l|}{ Weighted median MR } \\
\hline & & $\begin{array}{l}\beta(\mathrm{mmol} / \mathrm{L}) \\
(95 \% \mathrm{Cl})\end{array}$ & $P_{\mathrm{IVW}}$ & $P_{\mathrm{Het}}$ & $\begin{array}{l}\text { Intercept } \\
\text { (mmol/L) }\end{array}$ & $P_{\text {Intercept }}$ & $\beta$ (mmol/L) (95\% Cl) & $P_{\mathrm{wm}}$ \\
\hline $\begin{array}{l}\text { Preferentially drinking skim milk } \\
\text { (vs. any other milk type) }\end{array}$ & 3 & $0.050(0.032 ; 0.068)$ & $\begin{array}{l}3.8 \times \\
10^{-08}\end{array}$ & $\begin{array}{l}9.5 \times \\
10^{-03}\end{array}$ & $-1.6 \times 10^{-3}$ & 0.26 & $0.051(0.026 ; 0.075)$ & $\begin{array}{l}3.8 \times \\
10^{-05}\end{array}$ \\
\hline $\begin{array}{l}\text { Consuming tub margarine } \\
\text { (vs. no spread use) }\end{array}$ & 3 & $\begin{array}{l}-0.025(-0.034 ;- \\
0.015)\end{array}$ & $\begin{array}{l}1.3 \times \\
10^{-07}\end{array}$ & $\begin{array}{l}3.1 \times \\
10^{-05}\end{array}$ & $\begin{array}{l}-2.4 \times \\
10^{-3}\end{array}$ & 0.15 & $\begin{array}{l}-0.017(-0.032 ;- \\
0.001)\end{array}$ & 0.039 \\
\hline $\begin{array}{l}\text { Preferentially drinking milk } \\
\text { with a higher fat content* }\end{array}$ & 8 & $\begin{array}{l}-0.044(-0.063 ;- \\
0.024)\end{array}$ & $\begin{array}{l}1.5 \times \\
10^{-05}\end{array}$ & $\begin{array}{l}5.8 \times \\
10^{-03}\end{array}$ & $1.2 \times 10^{-4}$ & 0.87 & $\begin{array}{l}-0.053(-0.070 ;- \\
0.036)\end{array}$ & $\begin{array}{l}2.1 \times \\
10^{-09}\end{array}$ \\
\hline Dried fruit (pieces per day) & 25 & $\begin{array}{l}-0.018(-0.028 ;- \\
0.008)\end{array}$ & $\begin{array}{l}3.6 \times \\
10^{-04}\end{array}$ & 0.001 & $-6 \times 10^{-5}$ & 0.77 & $\begin{array}{l}-0.024(-0.035 ;- \\
0.013)\end{array}$ & $\begin{array}{l}3.1 \times \\
10^{-05}\end{array}$ \\
\hline
\end{tabular}

SNP number indicates the number of variants included in the instrumental variable

$P_{\text {Het }}$ indicates the level of heterogeneity observed between the variants included in the instrumental variable

*Preferred milk type (skimmed vs. semi-skimmed vs. full cream) as a quantitative variable

\section{Discussion}

Our previous study [10] concluded, using percent variance explained, that common genetic variants have a greater contribution to urate levels in the non-gout population than overall diet. Using attributable fraction measures, we arrive at the same conclusion, importantly also in a cohort of people with gout. Previously, the summed percent variance for the 30 genetic variants for urate levels was $8.7 \%$, considerably greater than the variance explained by the DASH diet [10]. Here, the summed PAFs for the 30 genetic variants was 141 to $146 \%$, considerably greater than that for following the DASH diet recommendations in cohorts 1 and 2 . Thus, empirically for $\mathrm{HU}$ at least, the different approaches of decomposition of variance and use of attributable fractions provide similar support for the greater relative role of common inherited genetic variation than overall diet in determining urate levels and HU. In the gout cohort, the attributable fractions for urate-lowering therapy were greater than for diet and BMI $<25 \mathrm{~kg} / \mathrm{m}^{2}$. Acknowledging the limitation that we were unable to build compliance, medication dose, and dosing to target into our models (which would contribute to under-estimating the effect of urate-
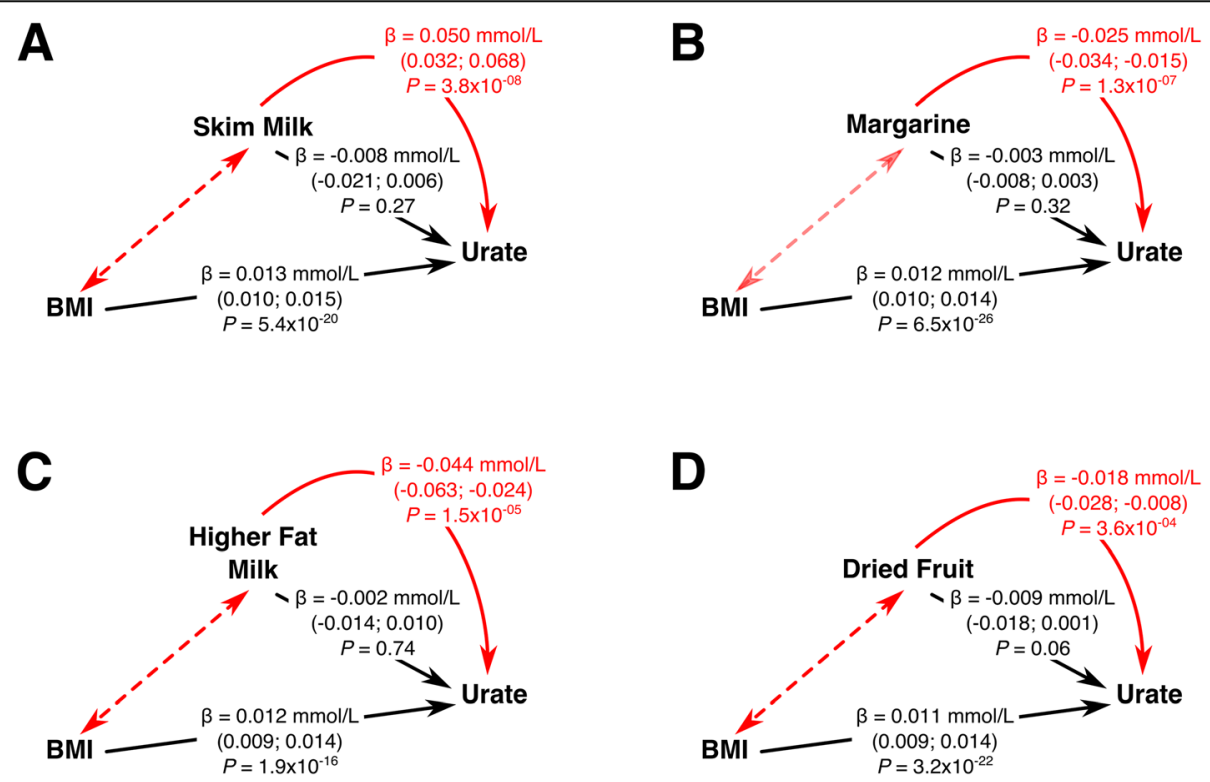

Fig. 2 Direct effect of dietary habits on serum urate levels, independent of BMI: multivariable Mendelian randomisation. The solid red arrow and values indicate the causal effect identified in the original inverse variance weighted MR; the dashed red arrow indicates the correlated pleiotropy between BMI and the dietary habit, influencing this original inverse variance weighted MR result; the black arrows and values indicate the causal effect independent of the other exposure variable. Beta values are in $\mathrm{mmol} / \mathrm{L}$. Figure 2a relates to results for preferentially drinking skim milk (vs. any other milk type); Fig. $2 b$ relates to results for consuming tub margarine (vs. no spread use) - the dashed red arrow is paler in this figure due to the lower confidence surrounding the correlated pleiotropy; Fig. $2 \mathrm{c}$ relates to results for preferentially drinking milk with a higher fat content; and Fig. $2 \mathrm{~d}$ relates to results for dried fruit consumption (pieces per day) 
lowering therapy), our data emphasise the importance of gold-standard clinical practice (urate-lowering therapy), to manage $\mathrm{HU}$ in gout patients. While weight reduction has established benefits, including to comorbidities in gout, our data demonstrate the greater impact of urate-lowering therapy in managing $\mathrm{HU}$ in gout.

While it is debatable whether public health efforts should be directed to primary prevention of HU, given the lack of evidence that $\mathrm{HU}$ is directly causal of conditions other than gout [44], there are two considerations that can be drawn. One, efforts would need to focus on interventions for which there is unequivocal evidence for a substantial impact to be made. This is not the case for the DASH diet (our AAF estimate in a multivariable model of the proportion of cases of $\mathrm{HU}$ prevented by following a DASH diet was only 6 to $7 \%$ ). Two, the proportion of cases of $\mathrm{HU}$ attributable to being overweight or obese from the population was 24 to $26 \%$ in the same model, only slightly more than the proportion attributable to SLC2A9 rs12498742 (22 to $24 \%$ ). It may seem incongruous to compare these exposures in the context of possible public health approaches to prevent primary $\mathrm{HU}$, given that it is not possible to prevent exposure to a common genetic variant. It is, however, possible to modify the impact of a genetic variant. The uricosuric drugs benzbromarone and probenecid inhibit the reuptake of filtered urate by GLUT9 (encoded by SLC2A9) [45]; thus, it is conceptually possible to target individuals with the rs12498742 urate-raising allele to improve excretion of urate and prevent HU. From a public health perspective, this is likely a more tractable intervention (in that it targets a single measurable exposure) than preventing obesity, which is caused by multiple environmental and genetic exposures that are not well understood.

That individual foods and estimates of dietary habits associate strongly with urate levels in observational data [10] does not necessarily translate into a clinically significant causal effect. It is interesting to compare association data of the DASH diet score [10] with data from a RCT of the effect of the DASH diet on serum urate levels [27] - the association data show a decrease of $0.023 \mathrm{mmol} / \mathrm{L}[0.38 \mathrm{mg} / \mathrm{dL}]$ between the least and most DASH-like diets in the US population [10], very similar to the $0.021 \mathrm{mmol} / \mathrm{L}[0.35 \mathrm{mg} / \mathrm{dL}]$ decrease when comparing the DASH diet with an 'average American diet' in the RCT [27]. In both cases, this is a relatively small change attributable to dietary habits and is reflective of the evidence presented here for a weak BMI-mediated causal relationship between diet and urate levels.

An incongruity is the apparent inconsistency between the two dairy-related MR analyses and results from RCTs [46-48]. Using MR as a complementary approach to investigate causality, we found only a small number of weak causal associations between dietary habits and serum urate. Interestingly, two of the significant causal associations represent opposing dietary habits, namely preferentially drinking skim milk or preferentially drinking milk with a higher fat content. The causal effects observed were consistent with these being opposing dietary habits, with skim milk consumption associating with increased urate, whilst consumption of higher-fat milk associated with decreased urate at an approximately equivalent effect size $(0.050 \mathrm{mmol} / \mathrm{L}[0.84 \mathrm{mg} / \mathrm{dL}]$ vs. $-0.044 \mathrm{mmol} / \mathrm{L}[-0.73 \mathrm{mg} / \mathrm{dL}]$, respectively). However, whilst these results are consistent with each other they are not consistent with prior studies of milk and dairy proteins in relation to urate levels. Observational studies have reported an inverse relationship between consumption of dairy products and serum urate levels $[7,10,49-$ 52]. Many of these observational studies do not separate dairy products into low and high fat content; however, those that do have found that this effect appears to be limited to consumption of skim or low-fat dairy products $[10,51]$. RCTs have supported these observational findings [46-48], in particular consumption of skim milk products acutely lowered serum urate levels by approximately $10 \%$ in 16 healthy adult men [46]. The apparent inconsistency between the MR and RCT results can be explained by the influence of BMI on the MR analysis. BMI appears to be a common upstream cause in the two dairy-related MR associations reported here, and these two dairy-related dietary habits are highly correlated with BMI, several measures of body fat and weight-loss related traits, including making major dietary changes to lose weight [26] (Table S4). It is plausible that the MR results reflect dietary recommendations given to individuals with a higher BMI (drink skim or low-fat milk), explaining the contradictory results seen here.

Our BMI genetic instrument explains more variance in type of milk consumed $(\sim 0.5 \%)$, than the milk type instruments do themselves (0.04 to $0.1 \%$ ) [26], highlighting an important limitation to these analyses. Genetic instruments for dietary habits likely explain a small fraction of phenotypic variance [26] or may be linked to diet through indirect mechanisms, potentially subjecting the MR analysis to bias towards the null, pleiotropy or confounding. While multiple MR approaches were used to address some of these pitfalls, future investigation using more biologically based genetic instruments for diet may illuminate previously undetectable causal relationships.

In the sample sets of European ancestry studied here, SLC2A9 rs12498742 had a considerably greater PAF than the $A B C G 2$ rs 2231142 variant (29 to $32 \%$ vs. $6 \%$, respectively (Table S2)). This is because of the 1.7-fold increased effect size of rs12498742 on serum urate levels and the increased prevalence of the urate-increasing allele $(77 \%$ vs. $11 \%)$ [35]. In contrast, in a Japanese study, 
the PAF for rs 2231142 was $29 \%$, compared to $19 \%$ for being overweight or obese [53], suggesting that for any primary prevention of $\mathrm{HU}$ in the Japanese population, targeting ABCG2 dysfunction would be a strategy to be considered. The rs2231142 risk allele frequency is $29 \%$ in the East Asian population compared to $9 \%$ in the European population. The authors of the Japanese study concluded that $A B C G 2$, at least, is a stronger risk factor for HU than other 'typical' environmental risk factors [53].

One limitation of the gout cohort analysis is the possibility of selection (collider) bias resulting from conditioning the sample set on gout ascertainment which would serve, when testing variables that are risk factors for gout per se, to bias effect sizes towards the null or even in an opposing direction [54]. This phenomenon likely explains the reduced (reversed for age) effect sizes for age, sex, BMI and diuretic exposure for each of risk of $\mathrm{HU}$ and change in serum urate levels and reduced variance explained, evidenced by non-overlapping 95\% CIs compared to cohorts 1 to 3 (Tables 2 and 3). For $S L C 2 A 9$, effect sizes and variance explained were lower in the gout cohort, but some confidence intervals were overlapping. However, for diet and alcohol, there was no difference in effect sizes (the 95\% CIs overlapped) suggesting that collider bias does not have a substantive effect on these estimates within the gout cohort. We note that the prevalence of healthy eating diet non-adherence was very similar between the UK Biobank Gout cohort and the equivalent non-gout cohort (cohort 3) indicating that diagnosis of gout did not change dietary behaviour. Selection bias will not influence our effect estimates for urate-lowering therapy; however, estimates for this exposure are likely inflated in the UK Biobank owing to healthy volunteer selection bias [55]. This likely leads to an over-estimate of effect size owing to exposure to urate-lowering therapy, because of a more compliant demographic. Our estimate of $\mathrm{OR}=20.2$ (Table 2) is considerably higher than a hazard ratio of 4.5 reported for achieving target urate in a gout cohort drawn from the UK primary care population [56]. While our estimate is not representative of the general population, it does indicate the possibility that the relative effect of uratelowering therapy on $\mathrm{HU}$ and serum urate levels is higher when compliance to urate-lowering therapy is increased.

\section{Conclusions}

In conclusion, we demonstrate using attributable fraction measures, that incorporate both the prevalence of exposure and effect size, the considerably greater attributable fraction of $\mathrm{HU}$ in the general population owing to common inherited genetic variants and BMI than to dietary exposure. The use of urate-lowering therapy in gout was the largest contributor to attributable fraction of HU. These findings are consistent with previous findings from the use of variance explained in the general population [10]. There is a weak causal effect between four dietary habits and urate levels, all mediated by BMI. Collectively, our findings refute the widely held perception that $\mathrm{HU}$ is primarily caused by diet [57-60].

\section{Supplementary Information}

The online version contains supplementary material available at https://doi. org/10.1186/s13075-021-02444-8.

\begin{abstract}
Additional file 1: Table S1. Sex-stratified population attributable and average attributable fractions for environmental and endogenous risk exposures for hyperuricaemia. Table S2. Population attributable and average attributable fractions for 30 genetic risk exposures for hyperuricaemia. Table S3. Causal effects of dietary habits on urate levels: Mendelian randomisation results. Table S4. Genetic variants comprising the four dietary habit instrumental variables.

Additional file 2: Text S1. Edited averageAF.R script for average attributable fraction confidence interval calculation. Figure S1. FISH plot of primary Mendelian randomisation analysis vs analysis including all ambiguous SNPS. Points in the upper right quadrant represent those with consistent effect directions and those in the lower left quadrant represent those with inconsistent effect directions. Nearly all of the points, especially the most significant points, are on the 45 degree line in the upper right quadrant.
\end{abstract}

\section{Abbreviations}

AAF: Average attributable fraction; ABCG2: ATP-binding cassette subfamily G member 2; ARIC: Atherosclerosis risk in communities; BMI: Body mass index; CARDIA: Coronary artery risk development in young adults; CHS: Cardiovascular heart study; DASH: Dietary approaches to stop hypertension; FHS: Framingham heart study; GLUT9: Glucose transporter 9; GWAS: Genome-wide association study; HU: Hyperuricemia; IVW: Inverse variance weighted; MR: Mendelian randomisation; PAF: Population attributable fraction; RCT: Randomised clinical trial; SLC2A9: Solute carrier family 2, member 9; ULT: Urate-lowering therapy

\section{Acknowledgements}

We thank the staff, participants and funding bodies of the ARIC, CARDIA, CHS, FHS and UK Biobank studies for their important contributions. We thank Hyon Choi for insightful discussion and anonymous peer reviewers for their constructive input.

This research has been conducted using the UK Biobank resource under application numbers 12611 and 11898 and Database of Genotypes and Phenotypes under application number 834. The ARIC study is carried out as a collaborative study supported by the National Heart, Lung, and Blood Institute contracts N01-HC-55015, N01-HC-55016, N01-HC-55018, N01-HC55019, N01-HC-55020, N01-HC-55021, N01-HC-55022, R01HL087641,

R01HL59367 and R01HL086694; National Human Genome Research Institute contract U01HG004402; and National Institutes of Health contract

HHSN268200625226C. Infrastructure was partly supported by Grant Number UL1RR025005, a component of the National Institutes of Health and NIH Roadmap for Medical Research. The FHS and the Framingham SHARe project are conducted and supported by the National Heart, Lung, and Blood Institute in collaboration with Boston University. The Framingham SHARe data used for the analyses described in this manuscript were obtained through the Database of Genotypes and Phenotypes. The CHS research reported in this article was supported by contract numbers N01-HC-85079, N01-HC85080, N01-HC-85081, N01-HC-85082, N01-HC-85083, N01-HC-85084, N01-HC85085, N01-HC-85086, N01-HC-35129, N01 HC-15103, N01 HC-55222, N01-HC75150, N01-HC-45133, N01-HC-85239 and HHSN268201200036C; grant numbers U01 HL080295 from the National Heart, Lung, and Blood Institute and R01 AG-023629 from the National Institute on Ageing, with additional contribution from the National Institute of Neurological Disorders and Stroke. A full list of principal CHS investigators and institutions can be found at www.chsnhlbi.org/pi.htm. The Coronary Artery Risk Development in Young Adults 
Study (CARDIA) is conducted and supported by the National Heart, Lung, and Blood Institute (NHLBI) in collaboration with the University of Alabama at Birmingham (N01-HC95095 and N01-HC48047), University of Minnesota (N01-HC48048), Northwestern University (N01-HC48049) and Kaiser Foundation Research Institute (N01-HC48050).

This manuscript was not prepared in collaboration with, nor approved by, investigators of the ARIC, CARDIA, CHS or FHS studies and does not necessarily reflect the opinions or views of these studies or their institutions or funding bodies.

\section{Authors' contributions}

RKGT, TJM, RJR, JBC and TRM contributed to the study design, analysed and interpreted the data and drafted the manuscript. All authors contributed to data analysis, interpretation and the manuscript. The authors read and approved the final manuscript.

\section{Funding}

This study was funded by the Health Research Council of New Zealand. The funder had no role in the design, execution and reporting of the study.

\section{Availability of data and materials}

All data used were publicly available. Derivative datasets generated during the current study are available from the corresponding author on reasonable request.

\section{Ethics approval and consent to participate}

Ethical approval for the UK Biobank participants was obtained from the North West Multi-Centre Research Ethics Committee (11/NW/0382). All participants gave written informed consent.

\section{Consent for publication}

Not applicable.

\section{Competing interests}

The authors declare that they have no competing interests.

\section{Author details \\ 'Department of Biochemistry, University of Otago, Dunedin, New Zealand. ${ }^{2}$ Programs in Metabolism and Medical \& Population Genetics, Broad Institute of MIT and Harvard, Cambridge, MA, USA. ${ }^{3}$ Diabetes Unit and Center for Genomic Medicine, Massachusetts General Hospital, Boston, MA, USA. ${ }^{4}$ Department of Medicine, Harvard Medical School, Boston, MA, USA. ${ }^{5}$ Division of Endocrinology and Center for Basic and Translational Obesity Research, Boston Children's Hospital, Boston, MA, USA. ${ }^{6}$ Department of Genetics, Harvard Medical School, Boston, MA, USA. ${ }^{7}$ Department of Medicine, Faculty of Medical Sciences, University of Auckland, Auckland, New Zealand. ${ }^{8}$ Department of Medicine, University of Otago Christchurch, Christchurch, New Zealand. ${ }^{9}$ Department of Mathematics and Statistics, University of Otago, Dunedin, New Zealand. ${ }^{10}$ Division of Clinical Immunology and Rheumatology, University of Alabama Birmingham, Birmingham, AL, USA.}

\section{Received: 6 September 2020 Accepted: 11 February 2021}

Published online: 04 March 2021

\section{References}

1. Boocock J, Leask M, Okada Y, Matsuo H, Kawamura Y, Shi Y, et al. Genomic dissection of 43 serum urate-associated loci provides multiple insights into molecular mechanisms of urate control. Hum Mol Genet. 2020;29:923-43.

2. Nakatochi M, Kanai M, Nakayama A, Hishida A, Kawamura Y, Ichihara S, et al. Genome-wide meta-analysis identifies multiple novel loci associated with serum uric acid levels in Japanese individuals. Comm Biol. 2019;2:115.

3. Tin A, Marten J, Kuhns V, Li Y, Wuttke M, Kirsten H, et al. Target genes, variants, tissues and transcriptional pathways influencing human serum urate levels. Nat Genet. 2019;51:1459-74.

4. Batt C, Phipps-Green AJ, Black MA, Cadzow M, Merriman ME, Topless R, et al. Sugar-sweetened beverage consumption: a risk factor for prevalent gout with SLC2A9 genotype-specific effects on serum urate and risk of gout. Ann Rheum Dis. 2014;73:2101-6.
5. Choi HK, Curhan G. Beer, liquor, and wine consumption and serum uric acid level: the Third National Health and Nutrition Examination Survey. Arthritis Rheum. 2004;51:1023-9.

6. Choi HK, Curhan G. Coffee, tea, and caffeine consumption and serum uric acid level: the Third National Health and Nutrition Examination Survey. Arthritis Rheum. 2007:57:816-21.

7. Choi HK, Liu S, Curhan G. Intake of purine-rich foods, protein, and dairy products and relationship to serum levels of uric acid: the Third National Health and Nutrition Examination Survey. Arthritis Rheum. 2005;52:283-9.

8. Rasheed H, Phipps-Green A, Topless R, Hollis-Moffatt JE, Harré Hindmarsh J, Franklin C, et al. Association of the lipoprotein receptor-related protein 2 gene with gout and non-additive interaction with alcohol consumption. Arthritis Res Ther. 2013;15:R177.

9. Choi HK, McCormick N, Lu N, Rai SK, Yokose C, Zhang Y. Population impact attributable to modifiable risk factors for hyperuricemia. Arthritis Rheumatol. 2020;72:157-65.

10. Major TJ, Topless RK, Dalbeth N, Merriman TR. Evaluation of the diet wide contribution to serum urate levels: meta-analysis of population based cohorts. BMJ. 2018;363:k3951.

11. McAdams DeMarco MA, Maynard JW, Baer AN, Gelber AC, Hunter Young J, Alonso A, Coresh J. Diuretic use, increased serum urate levels, and risk of incident gout in a population-based study of adults with hypertension: the Atherosclerosis Risk in Communities cohort study. Arthritis Rheum. 2012;64:121-9.

12. Savage PJ, Pressel SL, Curb JD, Schron EB, Applegate WB, Black HR, et al. Influence of long-term, low-dose, diuretic-based, antihypertensive therapy on glucose, lipid, uric acid, and potassium levels in older men and women with isolated systolic hypertension: the Systolic Hypertension in the Elderly Program. Arch Int Med. 1998;158:741-51.

13. Chen-Xu M, Yokose C, Rai SK, Pillinger MH, Choi HK. Contemporary prevalence of gout and hyperuricemia in the United States and decadal trends: the national health and nutrition examination survey, 2007-2016. Arthritis Rheumatol. 2019;71:991-9.

14. Ioannidis JP. The challenge of reforming nutritional epidemiologic research. JAMA. 2018;320:969-70.

15. Dalbeth N, Choi HK, Joosten LAB, Khanna PP, Matsuo H, Perez-Ruiz F, Stamp LK. Gout. Nat Rev Dis Prim. 2019;5:69.

16. Dalbeth N, Phipps-Green A, Frampton C, Neogi T, Taylor WJ, Merriman TR. Relationship between serum urate concentration and clinically evident incident gout: an individual participant data analysis. Ann Rheum Dis. 2018;77:1048-52.

17. Cox CL, Stanhope KL, Schwarz JM, Graham JL, Hatcher B, Griffen SC, et al. Consumption of fructose-but not glucose-sweetened beverages for 10 weeks increases circulating concentrations of uric acid, retinol binding protein-4, and gamma-glutamyl transferase activity in overweight/obese humans. Nutr Metab. 2012;9:68.

18. Le MT, Frye RF, Rivard CJ, Cheng J, MCFann KK, Segal MS, et al. Effects of high-fructose corn syrup and sucrose on the pharmacokinetics of fructose and acute metabolic and hemodynamic responses in healthy subjects. Metabolism. 2012;61:641-51.

19. Perez-Pozo S, Schold J, Nakagawa T, Sáchez-Lozada LG, Johnson RJ, López $\downarrow$. Excessive fructose intake induces the features of metabolic syndrome in healthy adult men: role of uric acid in the hypertensive response. Int J Obes. 2010;34:454-61.

20. Larsson SC, Burgess S, Michaëlsson K. Genetic association between adiposity and gout: a Mendelian randomization study. Rheumatology. 2018;57:2145-8.

21. Oikonen M, Wendelin-Saarenhovi M, Lyytikainen LP, Siitonen N, Loo B-M, Jula $A$, et al. Associations between serum uric acid and markers of subclinical atherosclerosis in young adults. The cardiovascular risk in Young Finns study. Atherosclerosis. 2012;223:497-503.

22. Palmer TM, Nordestgaard BG, Benn M, Tybjaerg-Hansen A, Davey Smith G, Lawlor DA, Timpson NJ. Association of plasma uric acid with ischaemic heart disease and blood pressure: Mendelian randomisation analysis of two large cohorts. BMJ. 2013;347:f4262.

23. Merino J, Dashti HS, Li SX, Sarnowski C, Justice AE, Graff M, et al. Genomewide meta-analysis of macronutrient intake of 91,114 European ancestry participants from the cohorts for heart and aging research in genomic epidemiology consortium. Mol Psych. 2019;24:1920-32.

24. Reed DR, Bachmanov AA, Beauchamp GK, Tordoff MG, Price RA. Heritable variation in food preferences and their contribution to obesity. Behav Genet. 1997;27:373-87.

25. Tanaka T, Ngwa JS, Van Rooij FJ, Carola Zillikens M, Wojczynski MK, FrazierWood C, et al. Genome-wide meta-analysis of observational studies shows 
common genetic variants associated with macronutrient intake. Am J Clin Nutr. 2013;97:1395-402.

26. Cole JB, Florez JC, Hirschhorn JN. Comprehensive genomic analysis of dietary habits in UK Biobank identifies hundreds of genetic associations. Nat Commun. 2020;11:1467.

27. Juraschek SP, Gelber AC, Choi HK, Appel LJ, Miller ER. Effects of the dietary approaches to stop hypertension (DASH) diet and sodium intake on serum uric acid. Arthritis Rheumatol. 2016;68:3002-9.

28. De Lorgeril M, Salen P, Martin J-L, Monjaud I, Delaye J, Mamelle N Mediterranean diet, traditional risk factors, and the rate of cardiovascular complications after myocardial infarction: final report of the Lyon Diet Heart Study. Circulation. 1999;99:779-85.

29. Wang DD, Leung CW, Li Y, Ding EL, Chiuve SE, Hu FB, Willett WC. Trends in dietary quality among adults in the United States, 1999 through 2010. JAMA Int Med. 2014:174:1587-95.

30. Zapata-Diomedi B, Barendregt JJ, Veerman JL. Population attributable fraction: names, types and issues with incorrect interpretation of relative risks. Br J Sports Med. 2018;52:212-3.

31. Cadzow M, Merriman TR, Dalbeth N. Performance of gout definitions for genetic epidemiological studies: analysis of UK Biobank. Arthritis Res Ther. 2017;19:181

32. Dalbeth D, Schumacher HR, Fransen J, Neogi T, Jansen TL, Brown M, et al. Survey definitions of gout for epidemiological studies: comparison with crystal identification as the gold standard. Arthritis Care Res. 2016;68:1894-8.

33. Bycroft C, Freeman C, Petkova D, Band G, Elliott LT, Sharp K, et al. The UK Biobank resource with deep phenotyping and genomic data. Nature. 2018; 562:203-9.

34. Loeb JN. The influence of temperature on the solubility of monosodium urate. Arthritis Rheum. 1972;15:189-92.

35. Köttgen A, Albrecht E, Teumer A, Vitart V, Krumsiek J, Hundertmark C, et al Genome-wide association analyses identify 18 new loci associated with serum urate concentrations. Nat Genet. 2013;45:145-54.

36. Rockhill B, Newman B, Weinberg C. Use and misuse of population attributable fractions. Am J Publ Health. 1998;88:15-9.

37. Rückinger $S$, von Kries R, Toschke AM. An illustration of and programs estimating attributable fractions in large scale surveys considering multiple risk factors. BMC Med Res Methodol. 2009;9:7.

38. Yavorska OO, Burgess S. MendelianRandomization: an R package for performing Mendelian randomization analyses using summarized data. Int J Epidemiol. 2017:46:1734-9.

39. Burgess S, Butterworth A, Thompson SG. Mendelian randomization analysis with multiple genetic variants using summarized data. Genet Epidemiol. 2013;37:658-65.

40. Bowden J, Davey Smith G, Burgess S. Mendelian randomization with invalid instruments: effect estimation and bias detection through Egger regression. Int J Epidemiol. 2015;44:512-25

41. Bowden J, Davey Smith G, Haycock PC, Burgess S. Consistent estimation in Mendelian randomization with some invalid instruments using a weighted median estimator. Genet Epidemiol. 2016:40:304-14.

42. Burgess S, Thompson SG. Multivariable Mendelian randomization: the use of pleiotropic genetic variants to estimate causal effects. Am J Epidemiol. 2015; 181:251-60.

43. Morrison J, Knoblauch N, Marcus JH, Stephens M, He X. Mendelian randomization accounting for correlated and uncorrelated pleiotropic effects using genome-wide summary statistics. Nat Genet. 2020;52:740-7.

44. Li X, Meng X, Timofeeva M, Tzoulaki I, Tsilidis KK, loannidis JP, et al. Serum uric acid levels and multiple health outcomes: umbrella review of evidence from observational studies, randomised controlled trials, and Mendelian randomisation studies. BMJ. 2017;357:j2376.

45. Dalbeth N, Stamp LK, Merriman TR. The genetics of gout: towards personalised medicine? BMC Med. 2017;15:108.

46. Dalbeth N, Wong S, Gamble GD, Horne A, Mason B, Pool B, et al. Acute effect of milk on serum urate concentrations: a randomised controlled crossover trial. Ann Rheum Dis. 2010;69:1677-82.

47. Garrel DR, Verdy M, PetitClerc C, Martin C, Brúle D, Hamet P. Milk-and soyprotein ingestion: acute effect on serum uric acid concentration. Am J Clin Nutr. 1991;53:665-9.

48. Ghadirian $P$, Shatenstein $B$, Verdy $M$, Hamet $P$. The influence of dairy products on plasma uric acid in women. Eur J Epidemiol. 1995;11:275-81.

49. Loenen HM, Eshuis H, Löwik MR, Schouten EG, Hulshof KF, Odink J, Kok FJ. Serum uric acid correlates in elderly men and women with special reference to body composition and dietary intake (Dutch nutrition surveillance system). J Clin Epidemiol. 1990:43:1297-303.

50. Ryu KA, Kang HH, Kim SY, Yoo MK, Seon Kim J, Haw Lee C, Ah WG. Comparison of nutrient intake and diet quality between hyperuricemia subjects and controls in Korea. Clin Nutr Res. 2014;3:56-63.

51. Zgaga L, Theodoratou E, Kyle J, Farrington AM, Agakov F, Tenesa A, et al. The association of dietary intake of purine-rich vegetables, sugar-sweetened beverages and dairy with plasma urate, in a cross-sectional study. PLoS One. 2012;7:e38123.

52. Zykova SN, Storhaug HM, Toft I, Chadban SJ, Jenssen TG, White SL. Crosssectional analysis of nutrition and serum uric acid in two Caucasian cohorts: the AusDiab Study and the Tromsø study. Nutr J. 2015;14:49.

53. Nakayama A, Matsuo H, Nakaoka H, Nakamura T, Nakashima H, Takada Y, et al. Common dysfunctional variants of ABCG2 have stronger impact on hyperuricemia progression than typical environmental risk factors. Sci Rep. 2014;4:5227.

54. Choi HK, Nguyen U-S, Niu J, Danaei G, Zhang Y. Selection bias in rheumatic disease research. Nat Rev Rheumatol 2014;10:403-412.

55. Fry A, Littlejohns TJ, Sudlow C, Doherty N, Adamska L, Sprosen T, et al. Comparison of sociodemographic and health-related characteristics of UK Biobank participants with those of the general population. Am J Epidemiol. 2017:186:1026-34

56. Rathod-Mistry T, Roddy E, Jordan K, Mallen CD, Blagojevic-Bucknall M. Longterm effect of allopurinol use on serum uric acid levels, mortality and comorbidities in gout patients: an electronic health record study. Rheumatol. 2019:S58:kez105.026.

57. Dalbeth N, Petrie KJ, House M, Chong J, Leung W, Chegudi R, et al. Illness perceptions in patients with gout and the relationship with progression of musculoskeletal disability. Arthritis Care Res. 2011;63:1605-12.

58. Duyck SD, Petrie KJ, Dalbeth N. "You don't have to be a drinker to get gout, but it helps": a content analysis of the depiction of gout in popular newspapers. Arthritis Care Res. 2016;68:1721-5.

59. Spaetgens B, Pustjens T, Scheepers LE, Janssens HJEM, van der Linden S, Boonen A. Knowledge, illness perceptions and stated clinical practice behaviour in management of gout: a mixed methods study in general practice. Clin Rheumatol. 2016;35:2053-61.

60. Spencer K, Carr A, Doherty M. Patient and provider barriers to effective management of gout in general practice: a qualitative study. Ann Rheum Dis. 2012;71:1490-5.

\section{Publisher's Note}

Springer Nature remains neutral with regard to jurisdictional claims in published maps and institutional affiliations.

Ready to submit your research? Choose BMC and benefit from:

- fast, convenient online submission

- thorough peer review by experienced researchers in your field

- rapid publication on acceptance

- support for research data, including large and complex data types

- gold Open Access which fosters wider collaboration and increased citations

- maximum visibility for your research: over $100 \mathrm{M}$ website views per year

At $\mathrm{BMC}$, research is always in progress.

Learn more biomedcentral.com/submissions 